\title{
Model for thermal conductivity of CNT-nanofluids
}

\author{
H E PATEL, K B ANOOP, T SUNDARARAJAN and SARIT K DAS* \\ Department of Mechanical Engineering, Indian Institute of Technology Madras, Chennai 600 036, India
}

\begin{abstract}
This work presents a simple model for predicting the thermal conductivity of carbon nanotube (CNT) nanofluids. Effects due to the high thermal conductivity of CNTs and the percolation of heat through it are considered to be the most important reasons for their anomalously high thermal conductivity enhancement. A new approach is taken for the modeling, the novelty of which lies in the prediction of the thermal behaviour of oil based as well as water based CNT nanofluids, which are quite different from each other in thermal characteristics. The model is found to correctly predict the trends observed in experimental data for different combinations of CNT nanofluids with varying concentrations.
\end{abstract}

Keywords. Thermal conductivity; nanofluid; carbon nanotube (CNT); nano-suspension; heat transfer.

\section{Introduction}

The trend of miniaturization has prompted heat transfer engineers to look for newer and innovative techniques for efficient cooling. Nanofluids are one of the potential technologies for heat transfer in future. The stable suspensions of nanoparticles (typically $<100 \mathrm{~nm}$ ) in liquids are called nanofluids; whereas suspension of carbon nanotube (CNT) in the liquid is called CNT nanofluid. Nanofluids are found to have very stable suspensions, without substantial sedimentation for a long time. They are found to eliminate most of the problems arising with slurries like sedimentation, clogging of small channels, erosion, excessive pressure drop etc. Moreover, the heat transfer capabilities of nanofluids are much enhanced as compared to base fluids. This makes them suitable for use in cooling of electronic equipments, lasers, fuel-cells, car radiators etc. The enhancement in thermal conductivity of nanofluids is unusually high and cannot be predicted by any of the conventional multiphase conductivity models like that of Maxwell-Garnett (1904). CNT nanofluids are found to be most effective in this regard, giving two orders of magnitude higher thermal conductivity enhancement compared to the usual slurries.

The greatest enhancement of thermal conductivity was observed in a subsequent study performed at ANL by Choi et al (2001). The nanofluid of multiwalled carbon nanotubes (MWCNTs) suspended in engine oil showed a phenomenal $150 \%$ increase in thermal conductivity with just $1 \%$ volume fraction of the nanotubes. This sudden jump in enhancement is interesting. With polymer nanotubes, similar enhancement was reported by Biercuk et al (2002). The reason for the abnormal rise of enhancement

\footnotetext{
*Author for correspondence (skdas@iitm.ac.in)
}

is yet to be explained but one can look at two facts. Firstly, the thermal conductivity of carbon nanotubes is very high $(\sim 2000 \mathrm{~W} / \mathrm{mK})$ and secondly, the nanotubes have a very high aspect ratio $(\sim 2000)$. We shall indicate the implications of the aspect ratio of the nanotubes when we consider the possible theories of thermal conductivity of nanofluids.

Assael et al (2005) measured thermal conductivity of multi walled as well as double walled CNTs. Thermal conductivity of MWCNTs of around $130 \mathrm{~nm}$ average diameter and $40 \mu \mathrm{m}$ average length was found to be $34 \%$ for $0.6 \%$ volume whereas that of double walled CNTs was found to be $8 \%$ for $1 \%$ volume suspension in water. Hwang et al (2006) also obtained similar results for MWCNT suspensions in water as well as ethylene glycol. Liu et al (2005) also measured thermal conductivity of MWCNTs of $20-50 \mathrm{~nm}$ in diameter. They observed an increase of $12.4 \%$ in the thermal conductivity of CNT suspension in ethylene glycol for $1 \%$ volume fraction whereas $30 \%$ enhancement in the CNT suspension in synthetic oil for $2 \%$ volume fraction.

In the last few years, attempts have been made to model the enhancement in thermal conductivity of CNT nanofluids by various assumptions like liquid layering, fractal theory etc. Xue (2005) modelled the thermal conductivity of CNT nanofluids using field factor approach, with a depolarization factor and an effective dielectric constant. The model is found to be working fairly well in predicting the thermal conductivities of CNT suspensions. Nan et al (2003) presented a simple formula for thermal conductivity enhancement in CNT composites which is derived from the Maxwell-Garnett (1904) model by the effective-medium approach. The model over-predicts the enhancement in the thermal conductivity of CNT suspensions when calculated with typical values of CNT thermal conductivities. Nan et al (2004) also developed a 
new model by incorporating interface thermal resistance with an effective-medium approach. However, the model needs the thermal resistance value at the surface of CNTs, which is difficult to get for different types of CNTs and their combinations with different solvents. Recently, Gao and Zhou (2006) presented another model, based on differential effective medium theory, derived from the MaxwellGarnett (1904) model. The model suggests a non-linear dependence of thermal conductivity of CNT nanofluids over volume concentration of the nanotubes, with slow increase at low concentrations and rapid at high concentrations.

There are some more models for the prediction of thermal conductivity of CNT nanofluids, but none of them conform to the experimental observations, for all the available experimental results.

The current work presents a model which will predict the thermal conductivity of CNT nanofluids. The existing model by Hemanth et al (2004), which predicts thermal conductivity of nanoparticles suspension, is improved here by removing the adjustable parameter used there. Two parallel paths of heat flow are considered viz. one through the conduction by liquid particles and another through the conduction by CNTs. Even at a very low volume fraction (1-2\%) of the CNTs, a continuous network of the CNTs is possible due to the very small diameter and very high aspect ratio. A TEM image of these nanofluids, as seen in figure 1, indicates that the CNTs are in a random network indeed. The four orders of magnitude high thermal conductivity of CNTs is the most significant contributor in the total heat transfer through the CNT nanofluid. The model is validated against available experimental data, for different base liquids.

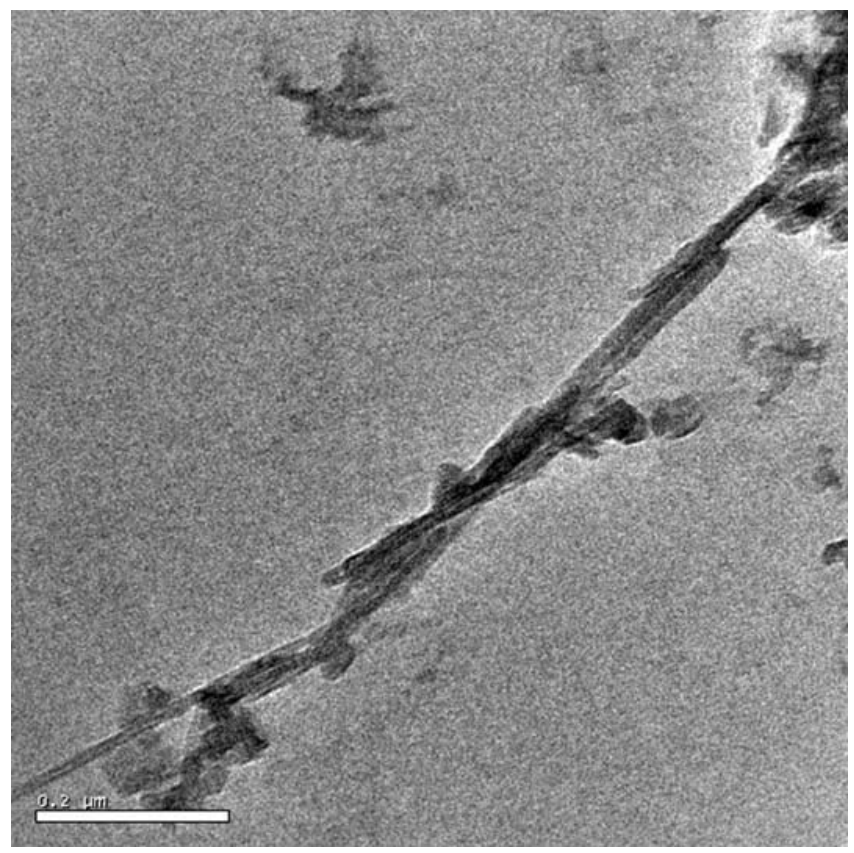

Figure 1. TEM image of CNTs.

\section{Present model}

The present model for thermal conductivity of CNT nanofluids is derived from Hemanth et al (2004), which is given for nanoparticle suspensions. The model considers two paths for heat to flow in a CNT nanofluid. One through the base liquid and the other one through the CNTs. These two paths are assumed to be in parallel to each other. Thus, two continuous media are considered here, participating in the conductive heat transfer. Usually, the aspect ratio of CNTs is very high (around 2000) and hence, a continuous net of CNTs available for heat transfer is a valid assumption. Thus, total heat transfer through nanofluid can be expressed as

$$
\begin{aligned}
& q=q_{1}+q_{\mathrm{s}}, \\
& q=-k_{1} A_{1}\left(\frac{d T}{d x}\right)_{1}-k_{\mathrm{s}} A_{\mathrm{s}}\left(\frac{\mathrm{d} T}{\mathrm{~d} x}\right)_{\mathrm{s}},
\end{aligned}
$$

where $A, k,(\mathrm{~d} t / \mathrm{d} x)$ denote the heat transfer area, thermal conductivity and temperature gradient of the respective media. Subscripts, 1 and s, denote quantities corresponding to the liquid medium and solid CNT medium, respectively. The liquid medium and the CNTs are assumed to be in local thermal equilibrium at each location, which gives

$$
\left(\frac{\mathrm{d} T}{\mathrm{~d} x}\right)_{1}=\left(\frac{\mathrm{d} T}{\mathrm{~d} x}\right)_{\mathrm{s}}=\left(\frac{\mathrm{d} T}{\mathrm{~d} x}\right)
$$

Thus, (1) can be written as

$$
q=-k_{1} A_{1}\left(\frac{\mathrm{d} T}{\mathrm{~d} x}\right)\left[1+\left(\frac{k_{\mathrm{s}} A_{\mathrm{s}}}{k_{\mathrm{l}} A_{1}}\right)\right] .
$$

Hemanth et al (2004) derived the expression for thermal conductivity enhancement in nanoparticle suspensions denoting the liquid and solid particle radii to be $r_{1}$ and $r_{\mathrm{s}}$ as well as volume fraction of the nanoparticles as $\varepsilon$ and the volume fraction of the liquid as $(1-\varepsilon)$.

$$
q=-k_{1} A_{1}\left(\frac{\mathrm{d} T}{\mathrm{~d} x}\right)\left[1+\frac{k_{\mathrm{s}} \varepsilon r_{1}}{k_{1}(1-\varepsilon) r_{\mathrm{s}}}\right]=-k_{\mathrm{eff}} A_{1}\left(\frac{\mathrm{d} T}{\mathrm{~d} x}\right),
$$

where the effective thermal conductivity is expressed as

$$
k_{\mathrm{eff}}=k_{1}\left[1+\frac{k_{\mathrm{s}} \varepsilon r_{1}}{k_{1}(1-\varepsilon) r_{\mathrm{s}}}\right] \text {. }
$$

The rate of heat transfer can be expressed in dimensionless form as

$$
q^{*}=\frac{q}{-k_{1} A_{1}\left(\frac{\mathrm{d} T}{\mathrm{~d} x}\right)}=1+\frac{k_{\mathrm{s}} \varepsilon r_{1}}{k_{\mathrm{l}}(1-\varepsilon) r_{\mathrm{s}}}=\frac{k_{\mathrm{eff}}}{k_{\mathrm{l}}},
$$

where the numerator represents the rate of heat transfer of the nanoparticle suspension, and the denominator is the rate 
of heat transfer in the absence of nanoparticles. However, Hemanth et al (2004) did not explain the term $r_{1}$, i.e. liquid particle size. It was left as an undefined parameter and for the purpose of calculation, it was accommodated in the constant ' $c$ ' used in next step of the model.

Here, we use the same expression (7) as derived by the stationary particle method developed by them for calculating the effective thermal conductivity of CNT nanofluids. The $r_{1}$ is taken as liquid molecular size and $r_{\mathrm{p}}$ as average diameter of the suspended CNTs. The application of this model with above assumptions and definitions is presented here for predicting the thermal conductivity enhancement of CNT nanofluids.

\section{Results and discussion}

The model shows linear dependence on CNT volume concentration and inverse dependence on the CNT diameter. Figure 2 shows the comparison of the model with the experimental data of Choi et al (2001). The enormous enhancement in the thermal conductivity of multiwalled CNT in oil nanofluid at very low volume fractions is predicted by the model very well. The average size of the multiwalled CNTs used is $25 \mathrm{~nm}$ in diameter and $50 \mu \mathrm{m}$ in length with around 30 annular layers. The experiments show an enhancement of $157 \%$ at just $1 \%$ medium and the Hamilton-Crosser (1962) model fails completely to predict such a high enhancement.

Also, the experimental data shows the linear dependence of CNT concentration on the effective thermal volume of the CNTs and $45 \%$ enhancement at $0.4 \%$ volume concentration. The classical model for solid medium suspended in liquid medium conductivity of nanofluid is predicted well by the model. The liquid particle size for the oil used for calculations is $3 \cdot 1 \AA$. Figure 3 shows the comparison of the model with the experimental data of Hwang et al (2006). The experiments show that the multiwalled CNTs suspended in water show quite less enhancement in the thermal conductivity of nanofluid as compared to that of oil based CNT nanofluid. The average size of the multiwalled CNTs used is $30 \mathrm{~nm}$ in diameter and $10-50 \mu \mathrm{m}$ in length. The experiments show an enhancement of $11.3 \%$ at $1 \%$ volume of the CNTs and around $4 \%$ enhancement at $0.25 \%$ volume concentration.

Although the enhancement is relatively less as compared to the CNT in oil nanofluid, still it is much higher as compared to the classical model predictions. The present model over-predicts it by a little, however, gives a reasonable idea about the order of enhancement. The liquid particle size for water used for calculations is $2 \AA$. The linear dependence of the thermal conductivity of CNT nanofluid on the CNT concentration is once again confirmed.

The success of the model is in predicting both, very high and very low enhancements in the thermal conductivity of CNT nanofluids. The success of the model for other types of CNT nanofluids may depend on the other factors as well such as aspect ratio or length of the CNTs, as it will play a major role in fulfilling the requirement of a continuous medium of CNTs. Also, the type of surfactant used, hydrophobic or hydrophilic nature of CNTs may play a role in terms of the surface thermal resistance.

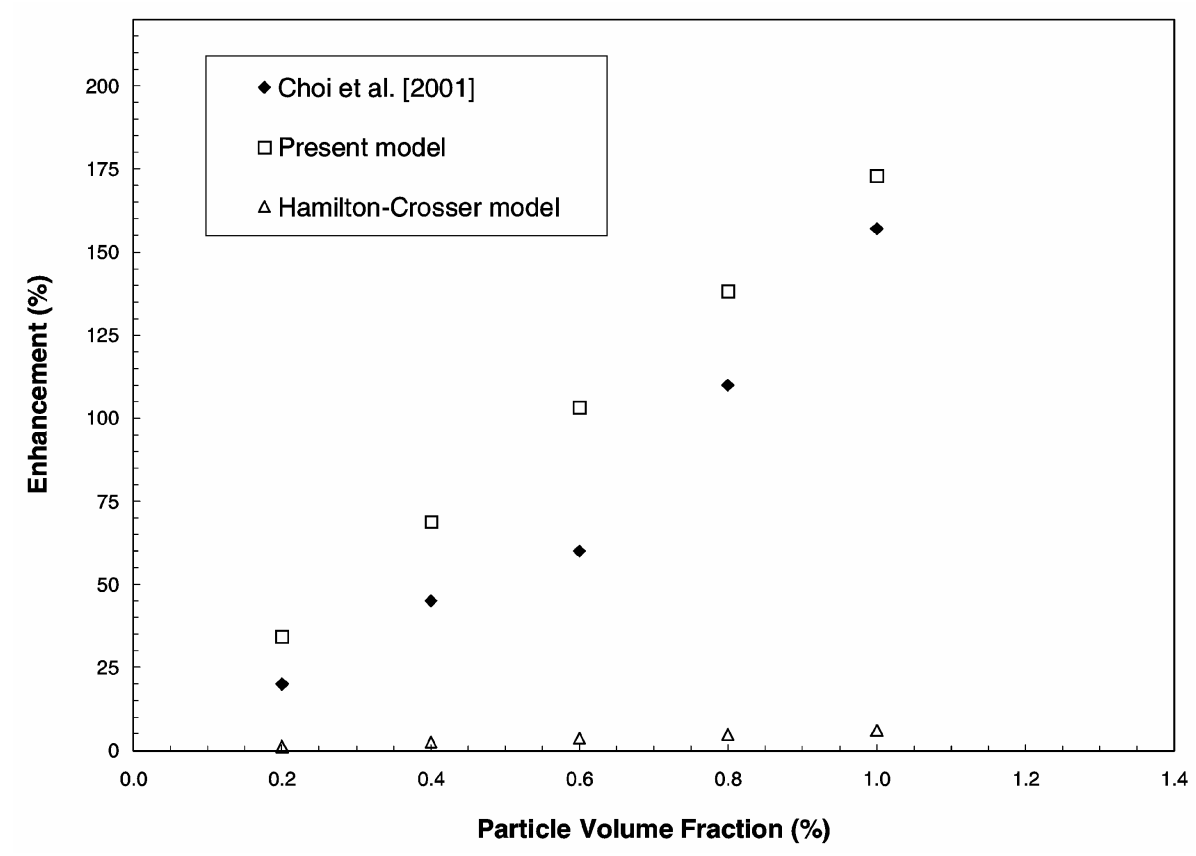

Figure 2. Comparison of model predictions with concentration variation for $\mathrm{CNT}$ in oil nanofluid. 


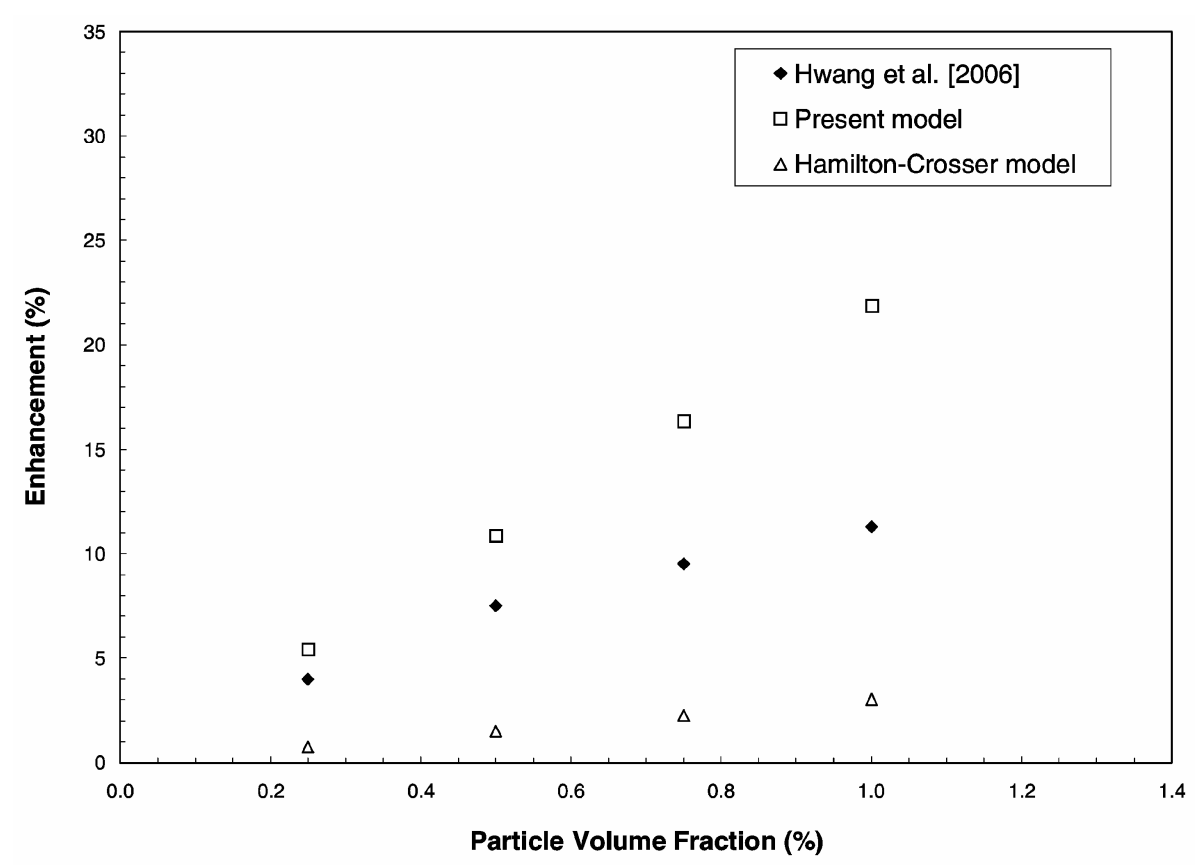

Figure 3. Comparison of model predictions with concentration variation for CNT in water nanofluid.

\section{Conclusions}

An existing theoretical model for predicting thermal conductivity of nanoparticle suspensions is improved so as to predict the thermal conductivity of CNT nanofluids. This is a completely new approach to model the thermal conductivity of CNT nanofluids. The model predictions successfully match the experimental data, varying over two orders of magnitude in their thermal conductivity enhancement values. The model predicts linear variation of the thermal conductivity of CNT nanofluid with volume concentration, which matches with the experimental data. It also predicts direct dependence on the thermal conductivity of CNTs and inverse dependence on the thermal conductivity of base liquid. Although the model is empirical in its derivation, however, it does not use any empirical constant.

\section{Nomenclature}

$A_{1}$, Heat transfer area per particle for conduction through liquid medium $\left(\mathrm{m}^{2}\right) ; A_{\mathrm{s}}$, surface area of nanoparticle $\left(\mathrm{m}^{2}\right)$; $d_{\mathrm{p}}$, average particle diameter $(\mathrm{m}) ; k_{\mathrm{l}}$, thermal conductivity of liquid medium $(\mathrm{W} / \mathrm{mK}) ; k_{\mathrm{s}}$, thermal conductivity of particle $(\mathrm{W} / \mathrm{mK}) ; q$, heat flux by conduction $\left(\mathrm{W} / \mathrm{m}^{2}\right) ; T$, temperature $(\mathrm{K})$.

\section{Subscripts}

eff, Effective; 1, liquid medium; s, solid medium.

\section{Greek symbols}

$\varepsilon$, Particle volume fraction.

\section{References}

Assael M J, Metaxa I N, Arvanitidis J, Christophilos D and Lioutas C 2005 Int. J. Thermophys. 26647

Biercuk M J, Llaguno M C, Radosavljevic M, Hyun J K, Johnson A T and Fischer J E 2002 Appl. Phys. Lett. 802767

Choi S U S, Zhang Z G, Yu W, Lockwood F E and Grulke E A 2001 Appl. Phys. Lett. 792252

Gao L and Zhou X F 2006 Phys. Lett. A348 355

Hamilton R L and Crosser O K 1962 I\&C Fundam. 1187

Hemanth K D, Patel HE, Rajeev K V R, Sundararajan T, Pradeep T and Das S K 2004 Phys. Rev. Lett. 93144301

Hwang Y J, Ahn Y C, Shin H S, Lee C G, Kim G T, Park H S and Lee J K 2006 Curr. Appl. Phys. 1068

Liu M S, Lin MCC, Huang I T and Wang C C 2005 Int. Comm. Heat Mass Transf. 321202

Maxwell-Garnett J C 1904 Philos. Trans. R. Soc. London, Series A 203385

Nan C W, Shi Z and Lin Y 2003 Chem. Phys. Lett. 375666

Nan C W, Liu G, Lin Y and Li M 2004 Appl. Phys. Lett. 853549

Xue Q Z 2005 Physica B368 302 